Journal of

Cardiology and Vascular Medicine

\title{
Impact of Three Dimensional In-Room Imaging (3DCA) in the Facilitation of Percutaneous Coronary Interventions
}

\author{
Marvin H. Eng ${ }^{1}$, Paul A. Hudson ${ }^{1}$, Andrew J. Klein ${ }^{2}$, S.Y. James Chen ${ }^{1}$, Michael S. Kim ${ }^{1}$, Bertron M. \\ Groves $^{1}$, John C. Messenger ${ }^{1}$, Onno Wink ${ }^{3}$, John D. Carroll ${ }^{1}$, and Joel A. Garcia ${ }^{1,4^{*}}$ \\ ${ }^{1}$ Division of Cardiology, University of Colorado Denver, Aurora, CO, USA \\ ${ }^{2}$ Division of Cardiology, John Cochran Veterans Affairs Medical Center, St.Louis, MO, USA \\ ${ }^{3}$ Philips Healthcare, Best, The Netherlands \\ ${ }^{4}$ Division of Cardiology, Denver Health Medical Center, Denver, CO, USA
}

${ }^{\star}$ Corresponding author: Joel A. Garcia, Associate Professor of Medicine, University of Colorado Denver, Leprino Office Building, Aurora, CO 80045, Tel: (720) 848-6508, Fax: (720) 848-7315, E-mail: joel.garcia@ucdenver.edu

Received Date: June 17, 2013 Accepted Date: August 16, 2013 Published Date: August 19, 2013

Citation: Joel A. Garcia (2013) Impact of Three Dimensional In-Room Imaging (3DCA) in the Facilitation of Percutaneous Coronary Interventions. J Cardio Vasc Med 1: 1-5.

\begin{abstract}
Introduction: Coronary angiography is a two-dimensional (2D) imaging modality and thus is limited in its ability to represent complex three-dimensional (3D) vascular anatomy. Lesion length, bifurcation angles/lesions, and tortuosity are often inadequately assessed using 2D angiography due to vessel overlap and foreshortening. 3D Rotational Angiography (3DRA) with subsequent reconstruction generates models of the coronary vasculature from which lesion length measurements and Optimal View Maps (OVM) defining the amount of vessel foreshortening for each gantry angle can be derived. This study sought to determine if 3DRA-assisted percutaneous coronary interventions resulted in improved procedural results by minimizing foreshortening and optimizing stent selection.

Methods: 26 patients with obstructive coronary artery disease were included. Rotational angiographic acquisitions were performed and a 3D model was generated from two images greater than $30^{\circ}$ apart. An optimal view map identifying the least amount of vessel foreshortening and overlap was derived from the 3D model. 3DRA derived and operator predicted optimal working view and stent lengths were compared.

Results: 3DRA assistance significantly reduced target vessel foreshortening when compared to operator's choice of working view for PCI $(2.99 \% \pm 2.96$ vs. $9.48 \% \pm 7.56, \mathrm{p}=0.0001)$. The operators concluded that 3DRA recommended better optimal view selection for PCI in 14 of $26(54 \%)$ total cases. In 9 (35\%) of 26 cases 3DRA assistance facilitated stent positioning. 3DRA based imaging prompted stent length changes in $4 / 26$ patients (15\%).

Conclusion: The use of 3DRA positively impacts the performance of percutaneous coronary interventions by optimizing working views through reductions in vessel foreshortening and overlap and assisting in stent positioning by improvements in stent and lesion measurements.
\end{abstract}

Keywords: Antioxidant system; Cadmium; Potassium deficiency; Rice

(C)2013 The Authors. Published by the JScholar under the terms of the Creative Commons Attribution License http://creativecommons.org/licenses/ by/3.0/, which permits unrestricted use, provided the original author and source are credited. 


\section{Introduction}

Coronary angiography remains the preferred modality for characterization of atherosclerotic coronary artery disease in contemporary cardiology. Because coronary angiography transforms a complex three-dimensional (3D) structure into a flat two-dimensional (2D) silhouette of the coronary lumen, however, the modality is inherently subject to numerous imaging limitations [1]. Most notably, the projection images may contain vessel foreshortening and overlap which may subsequently result in potential misjudging of critical vessel characteristics such as bifurcations, tortuosity, vessel and lesion size, lesion length, lesion eccentricity and angulation [1-3]. The traditional strategy used to minimize projection imaging inaccuracies is to acquire multiple views of a vessel segment for a more complete angiographic assessment. This assessment, however, comes at the cost of increased radiation, contrast exposure, and procedural time. Despite the additional information, optimal view selection is contingent on both individual operator skill and experience as well as unique patient anatomy and is often based purely on a trial-and-error technique. To overcome these obstacles, innovative angiographic techniques have been devised, most notably, rotational angiography (RA) with three-dimensional modeling (3DCA).

The need for optimal acquisitions and the recent availability of the rapid processing of angiographic data has led to the advent of 3D modeling technology. Chen and colleagues developed $3-\mathrm{D}$ coronary modeling approaches starting in the 1990's with subsequent commercialization through three vendors $[4,5]$. By using two angiographic views $>30^{\circ}$ apart, a $3 \mathrm{D}$ model of the coronary tree can be generated for subsequent display which allows for viewing from multiple perspectives, generation of an optimal view map, and advanced analysis of vessel segments [6]. Carroll and Chen subsequently developed the practical idea of utilizing $3 \mathrm{D}$ graphical data in map format to illustrate the interaction between gantry position and degree of vessel segment foreshortening and overlap [7]. Optimal Viewing Maps (OVM) identify optimal fluoroscopic viewing angles of specific coronary segments based on the degree of foreshortening and overlap [8,9]. The use of optimal view maps to facilitate angiography has been previously shown [5]. In addition, the use of both on-line and off-line 3DCA has also been previously validated along with its ability to estimate lesion length and predict stent length [10-12]. The implementation of in-room 3DCA to facilitate interventions is now possible with improved software and computer processing capabilities $[13,14]$. Despite these technological advancements, 3DCA has not yet been applied to Percutaneous Coronary Intervention (PCI) procedures in determining its direct impact on minimizing vessel foreshortening and overlap, as well as optimizing lesion length measurement and subsequent stent choice. To examine the potential benefits of 3DCA-assisted PCI, we prospectively evaluated the extent of vessel foreshortening, stent placement, and choice of stent length using 3DCA as compared to operator-projected optimal views and stent selection using Standard Angiography (SA).

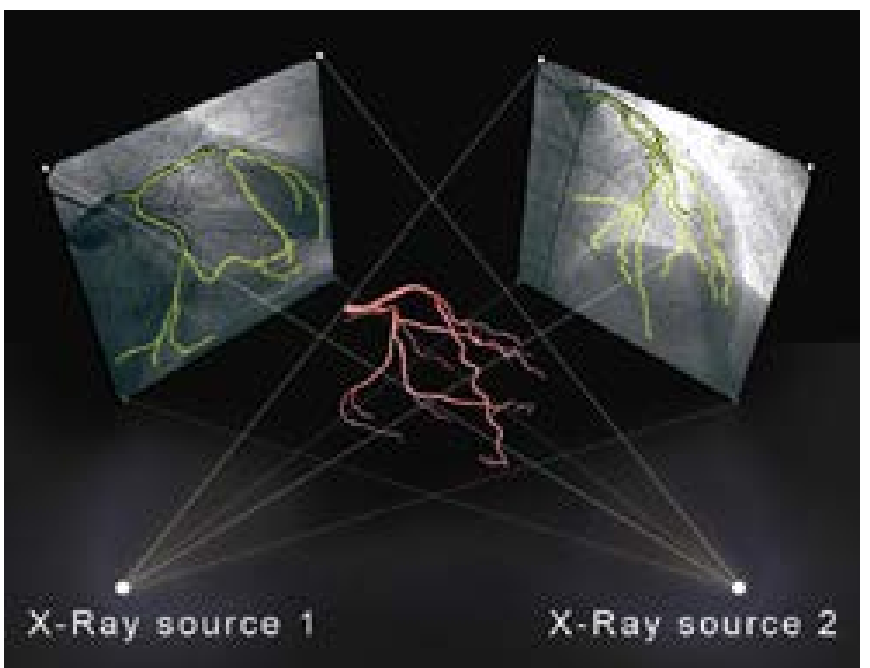

Figure 1: Three Dimensional coronary models reconstructed from two planar angiograms.

\section{Methods}

Patients undergoing angiography and subsequent PCI for obstructive coronary artery disease at the University of Colorado Hospital were included in this study. Exclusion criteria included renal insufficiency $(\mathrm{Cr}>2.0 \mathrm{mg} / \mathrm{dL})$, contrast allergy, or pregnancy. Patients were also excluded if the 3D modeling of their coronary tree was not feasible. The patients were enrolled with informed consent and this prospective study was approved by the Colorado Multiple Institutional Review Board.

\section{Study Protocol}

Images were acquired using a flat panel detector (Allura Xper FD20, Philips Healthcare, Best, The Netherlands) angiographic system. Cine runs were acquired at 30 frames per second in a standardized rotational format and transferred to a dedicated $3 \mathrm{D}$ workstation. Rotational angiographic (RA) acquisitions were performed beginning at $60^{\circ}$ right anterior oblique (RAO) to $60^{\circ}$ left anterior oblique (LAO) with $25^{\circ}$ of both cranial and caudal angulation respectively. The $\mathrm{C}$-arm rotates while acquiring images over a 4 second time span with an 8-12 ml injection of contrast medium. The resulting images were then transferred to the workstation automatically. Less than $45 \mathrm{sec}-$ onds are required for the images to transfer over to the workstation [11]. The 3DCA process and all vessel evaluations were completed by a trained physician and/or a clinical scientist familiar with the reconstruction system. The interventions were performed by board-certified interventional cardiologists. Due to conflict of interest, both Drs. Carroll and Chen did not participate in the execution of the protocol or data analysis.

3D modeling of the coronary vessels was performed by using a dedicated 3D workstation as previously described [6]. Briefly two images with full angiographic opacification are manually selected using the graphics interface in the $3 \mathrm{D}$ workstation, the vessels to be included in the model are manually identified in both views and a model is immediately generated. Subsequently the operator identifies the beginning and end of a lesion 
on the 3-D model. A color-coded map of the degree of foreshortening at all possible gantry positions is calculated and displayed. An optimal working view $\left(\right.$ True $\mathrm{View}^{\mathrm{R}}$ ) and a lesion length from the 3-D model are generated (True Length ${ }^{\mathrm{R}}$ ) while an estimated stent length is recommended by a clinical scientist or non-operator physician performing the evaluation. Image model generation takes less than 5 minutes. Simultaneously, operators blinded to the True View ${ }^{\mathrm{R}}$ and True Length ${ }^{\mathrm{R}}$ results select their preferred optimal working view and predicted stent length. Verification of both the operator selected and 3DRA assisted working views were performed and evaluated by the operators. Study protocol endpoints were 3DCA derived optimal view $\%$ foreshortening and stent length versus operator predicted views and stent length respectively (see Appendix).

\section{Statistics}

Continuous variables are presented as mean \pm SD. Differences between continuous variables were analyzed with the t-test.

\section{Results}

Of the fifty-four patients screened, 26 patients required PCI for significant lesions and were enrolled into the study. Complete 3-D reconstruction was unable to be accomplished in 3 patients $(6 \%)$ due to severe native vessel ostial disease resulting in study exclusion. A description of the 26 patients who underwent PCI is listed in Table 1. The target lesion was located in the left anterior descending artery (LAD) in 15/26 $(58 \%)$ cases, the right coronary artery (RCA) in $7 / 26(27 \%)$ cases, the Left Circumflex (LCx) artery in 3/26 (12\%) cases and the ramus intermedius in $1 / 26(3 \%)$ case. All patients successfully underwent $3 \mathrm{D}$ modeling with PCI and there were no complications.

\begin{tabular}{|l|l|}
\hline & $\mathrm{N}=26$ patients \\
\hline Mean Age & $59 \pm 10 \mathrm{yrs}$ \\
\hline Male & $80 \%$ \\
\hline CAD & $11(42 \%)$ \\
\hline Diabetes Mellitus & $5(19 \%)$ \\
\hline Hypertension & $20(77 \%)$ \\
\hline Dyslipidemia & $17(65 \%)$ \\
\hline Chronic Kidney Disease & $1(4 \%)$ \\
\hline Family History of CAD & $5(19 \%)$ \\
\hline Tobacco & $8(31 \%)$ \\
\hline Acute Coronary Syndrome & $7(27 \%)$ \\
\hline LVSD & $3(12 \%)$ \\
\hline LAD & $15(58 \%)$ \\
\hline RCA & $7(27 \%)$ \\
\hline LCx & $3(12 \%)$ \\
\hline Ramus & $1(4 \%)$ \\
\hline
\end{tabular}

$C A D=$ Coronary artery disease, $L V S D=$ Left ventricular systolic dysfunction $L A D-=L e f t$ anterior descending artery, $R C A=$ Right coronary artery, $L C X=$ Left circumflex artery.

Table 1: Study Patient Clinical Characteristics
3DCA assisted optimal view selection had less vessel foreshortening (FS) than operator selected optimal views $(2.8 \pm$ $2.7 \%$ vs. $9.2 \pm 7.6 \%$ FS, $\mathrm{p}=0.0001$ ) (Table 2 ). $3 \mathrm{DCA}$ derived optimal views were judged to have superior lesion visualization by highly trained, experienced, and board certified interventional cardiologists compared to operator selected optimal views in $54 \%$ of the cases. In the remaining 12 cases (46\%), 3DRA improved foreshortening but demonstrated more vessel overlap or recommended an infeasible gantry location (i.e. unobtainable gantry). 3DCA facilitated stent positioning in 9 cases (35\%) and impacted stent length in 4 cases (15\%) as it resulted in the operator changing the stent length based on a non-foreshortened measurement of the target vessel using 3DCA. Prospective operator and 3DCA stent length predictions were performed in 22 cases and were not found to be significantly different from the final stent length used $(\mathrm{p}=\mathrm{NS})$ (Table 3).

\begin{tabular}{|l|l|l|l|}
\hline Operator Selected & 3D Assisted & p-value & \\
\hline $9.2 \pm 7.6 \%$ & $2.8 \pm 2.7 \%$ & 0.0003 & Foreshortening \\
\hline
\end{tabular}

Table 2: Operator selected vs. 3D assisted optimal working view foreshortening.

\begin{tabular}{|l|l|l|}
\hline & Length in $\mathrm{mm}$ & P-value \\
\hline Actual stent length & $18.6 \pm 6.5$ & \\
\hline 3D assisted stent length & $19.4 \pm 6$ & 0.663 \\
\hline Operator stent length & $19.2 \pm 7$ & 0.74 \\
\hline
\end{tabular}

$3 D=$ three dimensional

Table 3: Comparison of operator predicted, 3D assisted and actual stent length used.

\section{Discussion}

This prospective evaluation of 3DRA-assisted PCI successfully demonstrates its practical application in PCI procedures to optimize angiographic working views and stent length choice. Although the interventional cardiologists participating in this single center trial each had $>10$ years experience, 3DCA-optimized angiographic working views in $54 \%$ of cases thereby illustrating its efficacy even in the hands of experienced operators. Furthermore, these changes in working views translated into assisting in stent placement and optimizing stent length in $35 \%$ and $15 \%$ of cases respectively, suggesting that a significant proportion of routine coronary interventions may not have optimal device dimension evaluations and subsequent placement. It is well known to the interventional community that the operator's stent length choice is often both generous in an effort to cover lesions adequately as well as constrained by the availability of stents in fixed length increments. Therefore, subtle differences in lesion length (3DCA vs. operator) may result in similar stent lengths. Measuring actual lesion length may have been a better marker for differences between the operator length and the 3DCA-assisted length. Overall, in an economic environment where rising healthcare costs need to be addressed, strategies to improve stent selection, decrease the risk of in-stent restenosis or side branch jailing due to longer stents, stent -positioning and equipment utilization are crucial [12]. 
A unique facet of this study was the routine use of RA for the initial imaging of the coronary vasculature which inherently provides many more images than standard angiography. Indeed, RA has been shown to improve PCI working view selection by providing a higher volume of information per angiogram $[15,16]$. This stands in contrast to SA wherein only 6-10 images are acquired in a diagnostic angiographic study. The use of RA therefore allowed the operators in this study the luxury of having a sizable anatomy survey, potentially resulting in an improved optimal view choice. It can be postulated that the use of standard fixed views may have resulted in operator selected optimal views exhibiting greater vessel foreshortening. Nevertheless, even with operators using RA, 3DCA-predicted working views still had statistically significantly less foreshortening and improved imaging in a majority of cases. A better reflection of the possible impact 3DCA may have in conventional cardiac catheterization labs would be a similar study using fixed angiographic views which more closely resembles the current standard. The software package used for the study supports 3DCA only with rotational acquisitions but the use of fixed angiographic views in performing 3DCA is being explored and currently incorporated in other $3 \mathrm{D}$ software packages [12].

The clinical validation of in-room image-processing tools such as 3DCA and optimal view maps is important since FDA approval of these tools does not require the presentation of any data on clinical experience and impact on clinical outcomes. While the technology of 3DRA and optimal view calculations has been well validated by the work of Chen and colleagues, this study is important in demonstrating how clinical care may be impacted $[4,5,7]$. This study was biased toward minimizing the impact of these tools on clinical decision-making since the study site, cardiologists, and staff have extensive experience in rotational angiography, 3-D modeling and reconstruction, and the impact of foreshortening on the assessment of lesion length and choice of stent size.

This study of 3DCA as an interventional tool bears several limitations. 3DCA reconstructs the major vessels and side branches but smaller vessel are not accounted for and those may contribute to significant vessel overlap obscuring important angiographic findings. This limitation can be overcome by completing the whole tree reconstruction. Gantry positions are constrained by body habitus and table position and 3DCA recommended views are not always necessarily practical. Another important parameter, vessel diameter was not evaluated as this study predated the addition of vessel sizing to the imaging software. Newer studies will include vessel size evaluations. The operators were allowed to use rotational angiography which may have resulted in better optimal view selection than the practice of standard fixed view angiography as detailed above. The impact of 3DCA as it related to operators without $3 \mathrm{D}$ or rotational experience remains a question worthy of further investigation. Lesion lengths may have been underestimated since they were determined through angiographic projections and not via intravascular ultrasound. Finally, most of the reconstructions were performed by a clinical scientist with no interventional background. It is possible that the reconstructions and gantry suggestions may have been improved by an interventionalist being allowed to review the images in-room and pick optimal gantry choices.

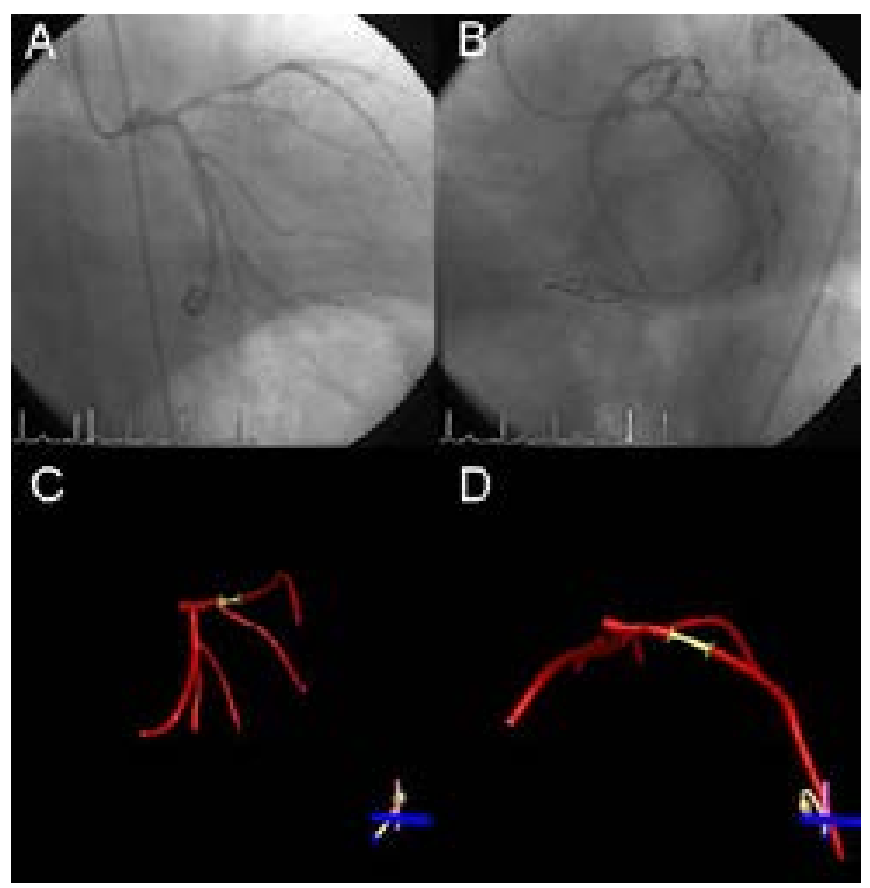

Figure 2: 3DRA analysis of the mid-LAD lesion.

A) and B) Initial RAO CAUD and LAO CAUD projections $>30^{\circ}$ in angulation utilized for 3D coronary model reconstruction. C) 3D model in operator selected view of RAO 1/CAUD 39 projection with 17\% foreshortening as described on the Optimal View Map (OVM). B) Rotated 3D reconstruction to RAO 45/CRAN 30 minimizing the foreshortening to $0 \%$ while enabling visualization of the first diagonal branch.

$3 D=$ three dimensional, $C R A N=$ cranial, $C A U D=$ caudal, $L A D=$ left anterior descending artery, $R A O=$ right anterior oblique

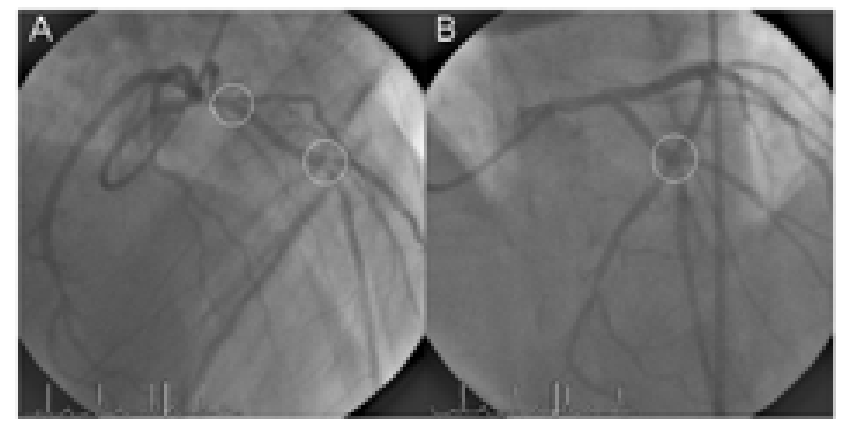

Figure 3: 3DRA assisted optimal view projection of the mid-LAD minimizing foreshortening and overlap.

A) 3DRA assisted projection of the post-PCI mid-LAD illustrating well separated diagonal branches (circles) with a minimally foreshortened mid-LAD. B) Prior operator selected view of the mid-LAD with an obscured first diagonal branch and overlapped second diagonal (circle). The impact of foreshortening length estimation is noted with a $17 \%$ difference length of the segment between the two diagonal branches in panel A versus panel B.

$3 D R A=$ three dimensional reconstruction, CRAN $=$ cranial, $L A D=$ left anterior descending artery, $P C I=$ percutaneous coronary intervention 
Initially the use of 3DCA was limited due to slow computer processing times and offline analysis. As computer graphics and processing times have improved this is a no longer a limitation. Use of 3DRA enables the generation of optimal view maps as a guide for working views for each vessel and has resulted in better view selection compared to operator selected views $[8,9]$. 3DCA reference vessel length and diameters have been shown to be similar to Quantitative Coronary Analysis (QCA), suggesting that procedural planning using 3DCA may be possible, even in complex lesions such as chronic total occlusions $[10,13]$. Post-stenting 3DRA analysis showed that improvements in stent selection and resource utilization may be achieved. Furthermore, the use of 3DCA in the analysis of stent conformational changes following an intervention have been shown and validated $[17,18]$. This study is the first prospective evaluation of 3DCA applied to a general population outside of individual cases [14]. The feasibility of applying 3DCA to a general population is suggested in this cohort and its role in cardiac catheterization laboratory may expand as efficiency in radiation exposure, contrast use and resource utilization is increasingly emphasized.

\section{References}

1) Topol EJ, Nissen SE (1995) Our preoccupation with coronary luminology. The dissociation between clinical and angiographic findings in ischemic heart disease. Circulation 92: 2333-2342.

2) Spears JR, Sandor T, Baim DS, Paulin S (1983) The minimum error in estimating coronary luminal cross-sectional area from cineangiographic diameter measurements. Cathet Cardiovasc Diagn 9: 119-128.

3) Scanlon PJ, Faxon DP, Audet AM, Carabello B, Dehmer GJ, et al. (1999) ACC/AHA guidelines for coronary angiography. A report of the American College of Cardiology/American Heart Association Task Force on practice guidelines (Committee on Coronary Angiography). Developed in collaboration with the Society for Cardiac Angiography and Interventions. J Am Coll Cardiol 33: 1756-824.

4) Chen SY, Hoffmann KR, Carroll JD (1996) Three-dimensional reconstruction of coronary artery tree based on biplane angiograms. Proc SPIE 2710: 103-114.

5) Chen SY, Schaefer D (2009) Three-dimensional coronary visualization, Part 1: modeling. In: Carroll JD, Chen SJ (eds) Advances in Coronary Angiography, An Issue of Cardiology Clinics. WB Saunders Co, Philadelphia.

6) Chen SJ, Carroll JD (2000) 3-D reconstruction of coronary arterial tree to optimize angiographic visualization. IEEE Trans Med Imaging 19: 318-336.

7) Carroll JD, Chen SY (2002) Method and Apparatus for Three Dimensional Reconstruction of Coronary Vessels from Angiographic Images and Analytical Techniques Applied Thereto. Patent No. US 6501848 B1.

8) Garcia JA, Movassaghi B, Casserly IP, et al. (2009) Determination of optimal viewing regions for X-ray coronary angiography based on a quantitative analysis of 3D reconstructed models. Int J Cardiovasc Imaging 25: 455-462.

9) Green NE, Chen SY, Hansgen AR, Messenger JC, Groves BM, Carroll JD (2005) Angiographic views used for percutaneous coronary interventions: a three-dimensional analysis of physician-determined vs. computer-generated views. Catheter Cardiovasc Interv 64: 451-459.

10) Agostoni P, Biondi-Zoccai G, Van Langenhove G, Kristoff Cornelis, Paul Vermeersch, et al. (2008) Comparison of Assessment of Native Coronary Arteries by Standard Versus Three-Dimensional Coronary Angiography. The American Journal of Cardiology 102: 272-279.
11) Chen SY, Carroll JD, Messenger JC (2002) Quantitative analysis of reconstructed 3-D coronary arterial tree and intracoronary devices. IEEE Trans Med Imaging 21: 724-740.

12) Gollapudi RR, Valencia R, Lee SS, Wong GB, Teirstein PS, et al (2007) Utility of three-dimensional reconstruction of coronary angiography to guide percutaneous coronary intervention. Catheterization and Cardiovascular Interventions 69: 479-482.

13) Dvir D, Assali A, Kornowski R (2008) Percutaneous coronary intervention for chronic total occlusion: Novel 3-dimensional imaging and quantitative analysis. Catheter Cardiovasc Interv 71: 784-789.

14) Garcia JA, Chen J, Hansgen A, Wink O, Movassaghi B, et al. (2007) Rotational angiography (RA) and three-dimensional imaging (3-DRA): an available clinical tool. Int J Cardiovasc Imaging 23: 9-13.

15) Garcia JA, Agostoni P, Green NE, Maddux JT, Chen J, et al. (2009) Rotational vs. standard coronary angiography: An image content analysis. Catheter Cardiovasc Interv 73: 753-761.

16) Maddux JT, Wink O, Messenger JC, Groves BM, Liao R, et al. (2004) Randomized study of the safety and clinical utility of rotational angiography versus standard angiography in the diagnosis of coronary artery disease. Catheter Cardiovasc Interv 62: 167-174.

17) Liao R, Chen SY, Messenger JC, Groves BM, Burchenal JE, et al. (2002) Four-dimensional analysis of cyclic changes in coronary artery shape. Catheter Cardiovasc Interv 55: 344-354.

18) Liao R, Green NE, Chen SY, Messenger JC, Hansgen AR, et al. (2004) Three-dimensional analysis of in vivo coronary stent - coronary artery interactions. Int J Cardiovasc Imaging 20: 305-313. 